\title{
Radiation Overexposure
}

National Cancer Institute

\section{Source}

National Cancer Institute. Radiation Overexposure. NCI Thesaurus. Code C72892.

Problem associated with excessive radiation emitted from radiological or diagnostic devices. 